\title{
CONVOLUTION OF HANKEL TRANSFORM AND ITS APPLICATION TO AN INTEGRAL INVOLVING BESSEL FUNCTIONS OF FIRST KIND
}

\author{
VU KIM TUAN
}

Institute of Mathematics, P.O.Box 6.31, Bo Ho, Hanoi, Vietnam

and

\section{MEGUMI SAIGO}

Department of Applied Mathematics, Fukuoka University, Fukuoka 814-01, Japan

(Received August 11, 1993)

\begin{abstract}
In the paper a convolution of the Hankel transform is constructed. The convolution is used to the calculation of an integral containing Bessel functions of the first kind.
\end{abstract}

Key Words and Phrases: Hankel transform, Bessel function, Convolution. 1991 AMS Subject Classification Codes: 33B20.

\section{Introduction}

The convolution of a modified Hankel transform, introduced in [4], has been studied in [1], [4] in classical sense and in [7] in a space of generalized functions. For an another modified Hankel transform the other convolution in some space of functions is obtained (see [5]).

The present paper is devoted to propose a definition of a convolution and to prove the convolution property in the classical sense of the following standard Hankel transform (see [6], [8])

$$
\mathcal{H}_{\nu}[f](x)=\int_{0}^{\infty} y J_{\nu}(y x) f(y) d y, \quad \operatorname{Re}(\nu)>-\frac{1}{2} .
$$

As one of its applications, a formula of infinite interval of a product of Bessel functions of the first kind is established.

\section{Convolution of Hankel Transform}

Set

$$
\begin{aligned}
h(x)=\frac{2^{1-3 \nu} x^{-\nu}}{\sqrt{\pi} \Gamma(\nu+1 / 2)} \int_{u+v>x,|u-v|<x}[ & {\left[{ }^{2}-(u-v)^{2}\right]^{\nu-1 / 2}\left[(u+v)^{2}-x^{2}\right]^{\nu-1 / 2} } \\
& \times(u v)^{1-\nu} f(u) g(v) d u d v, \quad x \in(0, \infty) .
\end{aligned}
$$

The function $h(x)$ is called the Hankel convolution of the function $f(x)$ with the function $g(x)$. It is easy to see that the convolution is a commutative operator of $f$ and $g$.

Let $L\left(R^{+} ; \mu(x)\right)$ be a class of integrable functions $f(x)$ with a weight $\mu(x)>0$ in $R^{+}=(0, \infty)$. The main aim of this section is to prove the following: 
Theorem. Let $\operatorname{Re}(\nu)>\frac{1}{2}$ and $f(x), g(x) \in L\left(R_{+} ; \sqrt{x}\right)$. Then the function $h(x)$ in $(2)$ cxists and there holds the convolution property

$$
\mathcal{H}_{\nu}[h](x)=x^{-\nu} \mathcal{H}_{\nu}[f](x) \mathcal{H}_{\nu}[g](x),
$$

where $\mathcal{J} \mathcal{C}_{\nu}$ is the Hankel transform (1).

Proof. It is well known $[6,(2.12 .42 .15)]$ that

$$
\begin{aligned}
\int_{0}^{\infty} t^{1-\nu} & J_{\nu}(x t) J_{\nu}(u t) J_{\nu}(v t) d t \\
& =\frac{2^{1-3 \nu}}{\sqrt{\pi} \Gamma(\nu+1 / 2)}(x u v)^{-\nu}\left[x^{2}-(u-v)^{2}\right]_{+}^{\nu-1 / 2}\left[(u+v)^{2}-x^{2}\right]_{+}^{\nu-1 / 2},
\end{aligned}
$$

where $\operatorname{Re}(\nu)>-\frac{1}{2}$ and

$$
\varphi_{+}(x)= \begin{cases}\varphi(x), & \varphi(x) \geqq 0, \\ 0, & \varphi(x)<0 .\end{cases}
$$

Since

$$
J_{\nu}(x)= \begin{cases}\sqrt{\frac{2}{\pi x}} \cos \left(x-\frac{\pi \nu}{2}-\frac{\pi}{4}\right)+O\left(x^{-3 / 2}\right), & (x \rightarrow+\infty), \\ O\left(x^{\nu}\right), & (x \rightarrow+0),\end{cases}
$$

(see [3]) it is easy to conclude that there exists such a positive number $C_{1}$ independent of $x \in(0, \infty)$ that

$$
\left|\sqrt{x} J_{\nu}(x)\right|<C_{1}, \quad x \in(0, \infty),
$$

and $x^{-\nu} J_{\nu}(x) \in L\left(R_{+}\right)$when $\operatorname{Re}(\nu)>\frac{1}{2}$. Therefore we have

$$
\begin{aligned}
\left|\int_{0}^{N} t^{1-\nu} J_{\nu}(x t) J_{\nu}(u t) J_{\nu}(v t) d t\right| & \leqq \frac{C_{1}^{2}}{\sqrt{u v}} \int_{0}^{N}\left|t^{-\nu} J_{\nu}(x t)\right| d t \\
& \leqq \frac{C_{1}^{2} x^{\operatorname{Re}(\nu)-1}}{\sqrt{u v}} \int_{0}^{\infty}\left|t^{-\nu} J_{\nu}(t)\right| d t \leqq \frac{C x^{\operatorname{Re}(\nu)-1}}{\sqrt{u v}}
\end{aligned}
$$

where $C$ is independent of $x, u, v$ and $N$. In particular, making use the formulas (2) and (4) with the help of the estimate (6) we have

$$
|h(x)| \leqq C x^{\operatorname{Re}(\nu)-1} \int_{0}^{\infty} \int_{0}^{\infty} \sqrt{u v}|f(u) g(v)| d u d v<\infty,
$$

since $f(x), g(x) \in L\left(R_{+} ; \sqrt{x}\right)$. Thus the function $h(x)$ in (2) exists. Furthermore, applying the Fubini theorem, we obtain

$$
\begin{aligned}
h(x) & =\int_{0}^{\infty} \int_{0}^{\infty} u v f(u) g(v) \int_{0}^{\infty} t^{1-\nu} J_{\nu}(x t) J_{\nu}(u t) J_{\nu}(v t) d t d u d v \\
& =\int_{0}^{\infty} t^{1-\nu} J_{\nu}(x t) \int_{0}^{\infty} \int_{0}^{\infty} u v J_{\nu}(u t) J_{\nu}(v t) f(u) g(v) d u d v d t \\
& =\int_{0}^{\infty} t J_{\nu}(x t) t^{-\nu} \mathcal{H}_{\nu}[f](t) \mathcal{H}_{\nu}[g](t) d t .
\end{aligned}
$$

Here we have used the existence of the Hankel transform $\mathcal{H}_{\nu}$ defined by (1) for functions from $L\left(R_{+} ; \sqrt{x}\right)$ (see [2], [8]). Moreover, we notice the fact

$$
\mathcal{H}_{\nu}[f](x)=O\left(x^{\nu}\right), \quad(x \rightarrow+0) \quad \text { for } f \in L\left(R_{+} ; \sqrt{x}\right)
$$

from [2, p. 74]. Therefore, if we set

$$
k(t)=t^{-\nu} \mathcal{H}_{\nu}[f](t) \mathcal{H C}_{\nu}[g](t),
$$


we have

$$
k(t)=O\left(t^{\prime \prime}\right), \quad(t \rightarrow+0) .
$$

On the other hand we have

$$
\left|\mathcal{J} C_{\nu}[f](t)\right| \leqq \frac{1}{\sqrt{t}} \int_{0}^{\infty}\left|\sqrt{u t} J_{\nu}(u t) \sqrt{u} f(u)\right| d u \leqq \frac{C}{\sqrt{t}}, \quad t \in(0, \infty),
$$

and therefore,

$$
k(t)=O\left(t^{-\nu-1}\right), \quad(t \rightarrow+\infty) .
$$

Since $\operatorname{Re}(\nu)>1 / 2$, from (9), (11) we conclude that $k(t) \in L\left(R_{+} ; \sqrt{t}\right)$. Therefore the formula (7) can be rewritten in the form

$$
h(x)=\mathcal{H C}_{\nu}[k](x) .
$$

Hence, by using the inversion formula of the Hankel transform in the class $L\left(R_{+} ; \sqrt{x}\right)$ (see [2], [8]):

$$
\mathcal{H}_{\nu}\left[\mathcal{H}_{\nu}[k]\right](x)=k(x),
$$

we obtain

$$
k(x)=\mathcal{H}_{\nu}[h](x) .
$$

As $k(x)$ has the form (8), the formula (13) coincides with the formula (3). Thus the theorem is proved.

\section{Application}

As an application of Theorem we consider the integral

$$
f_{y_{1}, y_{n}}^{\nu_{0}, \nu_{1}, \nu_{n}}\left(a_{0}, a_{1}, \cdots, a_{n}\right)=\int_{0}^{\infty} t^{\nu_{0}+1} J_{\nu_{0}}\left(a_{0} t\right) \prod_{j=1}^{n}\left(t^{2}+y_{\jmath}^{2}\right)^{-\nu_{\jmath} / 2} J_{\nu_{j}},\left(a, \sqrt{t^{2}+y_{j}^{2}}\right) d t
$$

with $a_{\jmath}>0(j=0,1, \cdots, n)$ and $\operatorname{Re}\left(y_{\jmath}\right) \geqq 0(j=1,2, \cdots, n)$. We will prove that

$$
f_{\mathbf{y}_{1}, \nu_{1}, \cdots, y_{n}}^{\nu_{0_{n}}, \nu_{n}}\left(a_{0}, a_{1}, \cdots, a_{n}\right)=0
$$

when

$$
a_{0}>a_{1}+\cdots+a_{n} \text { and } \frac{1}{2}<\operatorname{Re}\left(\nu_{0}\right)<\sum_{j=1}^{n} \operatorname{Re}\left(\nu_{\jmath}\right)+\frac{n-3}{2} .
$$

We know that it is valid for $n=1$ (see $[6,(2.12 .31 .1)]$ for the case $\operatorname{Re}\left(y_{1}\right)=0$, and $[6,(2.12 .35 .12)]$ for the case $\left.\operatorname{Re}\left(y_{1}\right)>0\right)$. Suppose that it is valid for every $k \leqq n$. We have to prove it for the case $k=n+1$. Put

$$
g_{y_{1}, \cdots, y_{n}}^{\nu_{0}, \nu_{1}, \cdots, \nu_{n}}\left(t, a_{1}, \cdots, a_{n}\right)=t^{\nu_{0}} \prod_{j=1}^{n}\left(t^{2}+y_{j}^{2}\right)^{-\nu, / 2} J_{\nu},\left(a, \sqrt{t^{2}+y_{j}^{2}}\right) .
$$

By using (5) we have

$$
\begin{aligned}
& g_{y_{1}, \cdots, y_{n}}^{\nu_{0}, \nu_{1}, \cdots, \nu_{n}}\left(t, a_{1}, \cdots, a_{n}\right)=O\left(t^{\nu_{0}}\right), \quad(t \rightarrow+0) \\
& =O\left(t^{\nu_{0}-\nu_{1}-}-\nu_{n}-n / 2\right), \quad(t \rightarrow+\infty) .
\end{aligned}
$$

Suppose that

$$
\frac{1}{2}<\operatorname{Re}\left(\nu_{0}\right)<\sum_{j=1}^{n} \operatorname{Re}\left(\nu_{\jmath}\right)+\frac{n-3}{2} .
$$

Then from (14) we conclude that $g_{y_{1}, \cdots, y_{n}}^{\nu_{0}, \nu_{1}, \cdots, \nu_{n}}\left(t, a_{1}, \cdots, a_{n}\right) \in L\left(R_{+} ; \sqrt{t}\right)$. Therefore, by using the formula (10) we obtain

$$
\mathcal{H} \mathcal{L}_{\nu_{0}}\left[g_{y_{1}, \cdot, y_{n}}^{\nu_{0}, \nu_{1}, \cdots, \nu_{n}}\left(t, a_{1}, \cdots, a_{n}\right)\right](x)=O\left(\frac{1}{\sqrt{x}}\right), \quad(x \rightarrow+0, x \rightarrow+\infty) .
$$

Since

$$
\mathcal{H}_{\nu_{0}}\left[g_{y_{1},, y_{n}}^{\nu_{0}, \nu_{1}, \nu_{n}}\left(t, a_{1}, \cdots, a_{n}\right)\right](x)=f_{y_{1}, \cdots, y_{n}}^{\nu_{0}, \nu_{1}, \nu_{n}}\left(x, a_{1}, \cdots, a_{n}\right)
$$


the formula (1.5) can be read as

$$
f_{y_{1},, y_{n}}^{\nu_{0}, \nu_{1}, \nu_{n}}\left(x, a_{1}, \cdots, a_{n}\right)=O\left(\frac{1}{\sqrt{x}}\right), \quad(x \rightarrow+0, x \rightarrow+\infty) .
$$

But by the assumption we have

$$
f_{y_{1}, y_{n}}^{\nu_{0}, \nu_{1}, \nu_{n}}\left(x, a_{1}, \cdots, a_{n}\right)=0
$$

when $x>a_{1}+\cdots+a_{n}$. Therefore

$$
f_{y_{1}, y_{n}}^{\nu_{0}, \nu_{1}, \nu_{n}}\left(x, a_{1}, \cdots, a_{n}\right) \in L\left(R_{+} ; \sqrt{x}\right)
$$

and by (12)

$$
\mathcal{H}_{\nu_{0}}\left[f_{y_{1}, y_{n}}^{\nu_{0}, \nu_{1}, \nu_{n}}\left(x, a_{1}, \cdots, a_{n}\right)\right](t)=g_{y_{1},, y_{n}}^{\nu_{0}, \nu_{1}, \nu_{n}}\left(t, a_{1}, \cdots, a_{n}\right) .
$$

Analogously, we have

$$
f_{y_{n+1}}^{\nu_{0}, \nu_{n+1}}\left(x, a_{n+1}\right) \in L\left(R_{+} ; \sqrt{x}\right)
$$

and

under the conditions

$$
\mathcal{H}_{\nu_{0}}\left[f_{y_{n+1}}^{\nu_{0}, \nu_{n+1}}\left(x, a_{n+1}\right)\right](t)=g_{y_{n+1}}^{\nu_{0}, \nu_{n+1}}\left(t, a_{n+1}\right)
$$

$$
\frac{1}{2}<\operatorname{Re}\left(\nu_{0}\right)<\operatorname{Re}\left(\nu_{n+1}\right)-1
$$

Since

$$
g_{y_{1}, y_{n+1}}^{\nu_{0}, \nu_{1}, \nu_{n+1}}\left(t, a_{1}, \cdots, a_{n+1}\right)=t^{-\nu_{0}} g_{y_{1}, y_{n}}^{\nu_{0}, \nu_{1}, y_{n} \nu_{n}}\left(t, a_{1}, \cdots, a_{n}\right) g_{y_{n+1}}^{\nu_{0}, \nu_{n+1}}\left(t, a_{n+1}\right)
$$

then by using the theorem we obtain

$$
\begin{gathered}
f_{y_{1}, y_{n+1}}^{\nu_{0}, \nu_{1}, \nu_{n+1}}\left(x, a_{1}, \cdots, a_{n+1}\right)=\int_{0}^{\infty} t J_{\nu_{0}}(x t) t^{-\nu_{0}} g_{y_{n+1}}^{\nu_{0}, \nu_{n+1}}\left(t, a_{n+1}\right) g_{y_{1}, \cdots, y_{n}}^{\nu_{0}, \nu_{1}, \nu_{n}}\left(t, a_{1}, \cdots, a_{n}\right) d t \\
=\mathcal{H}_{\nu_{0}}\left[t^{-\nu_{0}} \mathcal{H}_{\nu_{0}}\left[f_{y_{n+1}}^{\nu_{0}, \nu_{n+1}}\left(y, a_{n+1}\right)\right](t) \cdot \mathcal{H}_{\nu_{0}}\left[f_{y_{1}, \cdots, y_{n}}^{\nu_{0}, \nu_{1}, \cdots, \nu_{n}}\left(y, a_{1}, \cdots, a_{n}\right)\right](t)\right](x) \\
=\frac{2^{1-3 \nu_{0}} x^{-\nu_{0}}}{\sqrt{\pi} \Gamma\left(\nu_{0}+1 / 2\right)} \iint_{u+v>x,|u-v|<x}\left(x^{2}-(u-v)^{2}\right)^{\nu_{0}-1 / 2}\left((u+v)^{2}-x^{2}\right)^{\nu_{0}-1 / 2}(u v)^{1-\nu_{0}} \\
\times f_{y_{1}, \cdots, y_{n}}^{\nu_{0}, \nu_{1}, \cdots, \nu_{n}}\left(u, a_{1}, \cdots, a_{n}\right) f_{y_{n+1}}^{\nu_{0}, \nu_{n+1}}\left(v, a_{n+1}\right) d u d v
\end{gathered}
$$

Since

$$
f_{y_{1}, \cdots, y_{n}}^{\nu_{0}, \nu_{1}, \nu_{n}}\left(u, a_{1}, \cdots, a_{n}\right)=0 \text { when } \quad u>a_{1}+\cdots+a_{n}
$$

and

provided that

$$
f_{y_{n+1}}^{\nu_{0}, \nu_{n+1}}\left(v, a_{n+1}\right)=0 \quad \text { when } \quad v>a_{n+1}
$$

$$
\frac{1}{2}<\operatorname{Re}\left(\nu_{0}\right)<\sum_{j=1}^{n} \operatorname{Re}\left(\nu_{\jmath}\right)+\frac{n-3}{2}, \quad \operatorname{Re}\left(\nu_{0}\right)-\operatorname{Re}\left(\nu_{n+1}\right)<-1,
$$

we conclude from (16) that

$$
f_{y_{1}, y_{n+1}}^{\nu_{0}, \nu_{1},, \nu_{n+1}}\left(x, a_{1}, \cdots, a_{n+1}\right)=0 \quad \text { when } \quad x>a_{1}+\cdots+a_{n+1}
$$

under (17).

The formula (18) can be analytically continued to the domain

$$
-1<\operatorname{Re}\left(\nu_{0}\right)<\sum_{\jmath=1}^{n+1} \operatorname{Re}\left(\nu_{\jmath}\right)+\frac{n-3}{2} .
$$

Thus we have proved 
Corollary. Let

$$
-1<\operatorname{Re}\left(\nu_{0}\right)<\sum_{j=1}^{n} \operatorname{Re}\left(\nu_{\jmath}\right)+\frac{n-3}{2}, \quad a,>0(j=1, \cdots, n)
$$

with

$$
a_{0}>a_{1}+\cdots+a_{n}
$$

and

$$
\operatorname{Re}\left(y_{\jmath}\right) \geqq 0(j=1, \cdots, n) .
$$

Then

$$
\int_{0}^{\infty} t^{\nu_{0}+1} J_{\nu_{0}}\left(a_{0} t\right) \prod_{j=0}^{n}\left(t^{2}+y_{\jmath}^{2}\right)^{-\nu, / 2} J_{\nu}\left(a, \sqrt{t^{2}+y_{j}^{2}}\right) d t=0
$$

The formula (19) is a generalization of the formulae (2.12.44.7) (the case $y_{1}=\cdots=y_{n}=0$ ) and (2.12.44.8) (the case $\left.\operatorname{Re}\left(y_{1}\right)>0, \cdots, \operatorname{Re}\left(y_{n}\right)>0\right)$ in [6].

Acknowledgement. The work of the first author was supported, in part, by the National Basic Research Program in Natural Sciences, Vietnam, and hy the Alexander von Humboldt Foundation.

\section{References}

[1] F.M. Cholewinski: Hankel Convolution Complex Inversion Theory, Mem. Amer. Math. Soc. Vol. 58, 1965.

[2] V.A. Ditkin and A.P. Prudnikov: Integral Transforms and Operational Calculus, Pergamon Press, Oxford-London-Edinburgh-New York-Paris-Frankfurt, 1965.

[3] A. Erdélyi, W. Magnus, F. Oberhettinger and R.P. Soni: Higher Transcendental Functions, Vol. 2, McGraw-Hill, New York-Toronto-London, 1953.

[4] I.I. Hirschman, Jr.: Variation diminishing Hankel transforms, J. Analyse Math. 8(1960/61), 307-336.

[5] Nguyen Thanh Hai and S.B. Yakubovich: The Double Mellin-Barnes Type Integrals and Their Applications to Convolution Theory, World Scientific, Singapore-New Jerser-London-Hong Kong, 1992.

[6] A.P. Prudnikov, Yu.A. Brychkov and O.I. Marichev: Integrals and Series, Vol.2, Special Functions, Gordon \& Breach, New York-London-Paris-Tokyo, 1986.

[7] J. de Sousa Pinto: A generalized Hankel convolution, SIAM J. Math. Anal. 16 (1985), 1335-1346.

[8] E.C. Titchmarsh: Introduction to the Theory of Fourier Integrals, Oxford Univ. Press, Oxford, 1948. 


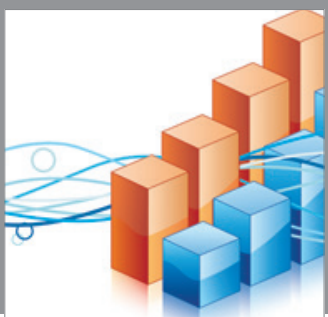

Advances in

Operations Research

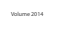

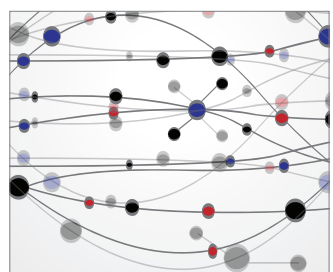

\section{The Scientific} World Journal
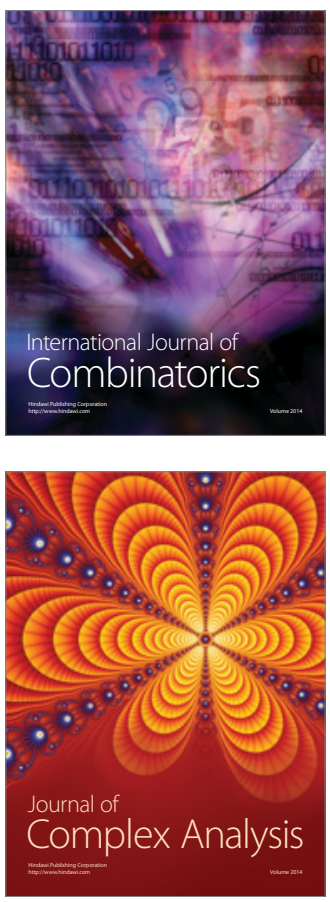

International Journal of

Mathematics and

Mathematical

Sciences
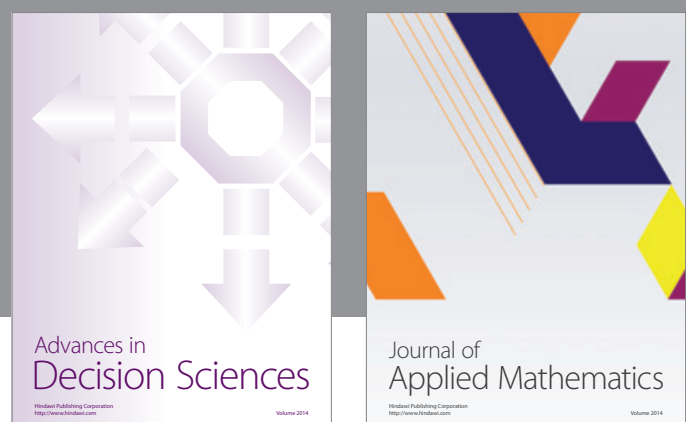

Journal of

Applied Mathematics
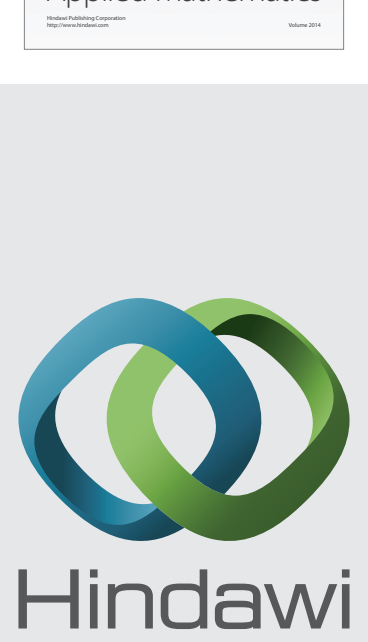

Submit your manuscripts at http://www.hindawi.com
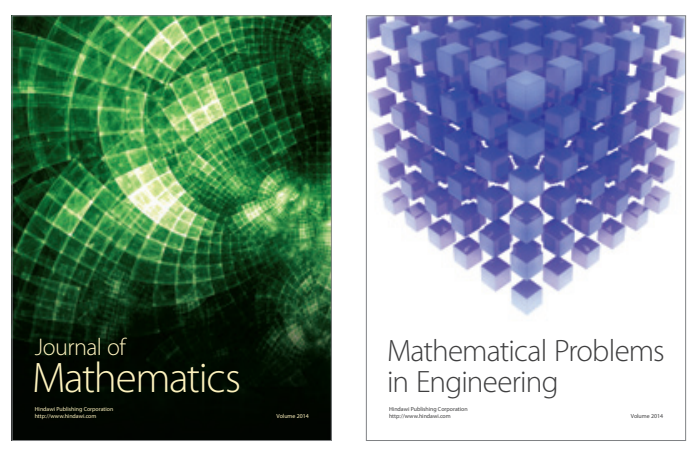

Mathematical Problems in Engineering
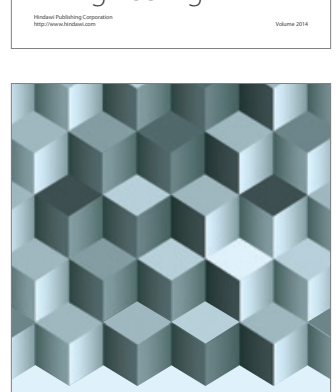

Journal of

Function Spaces
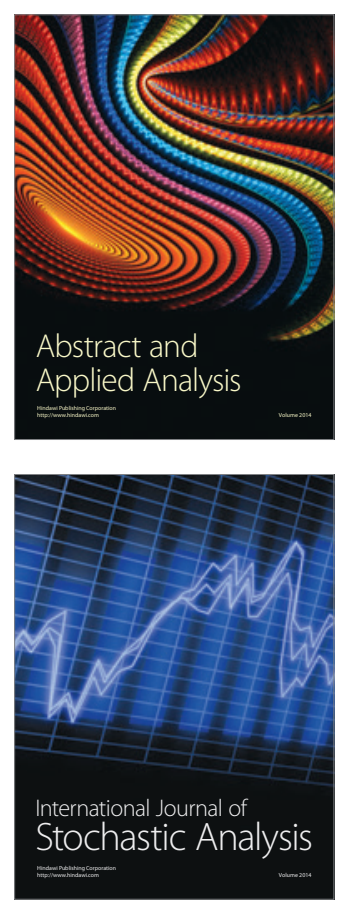

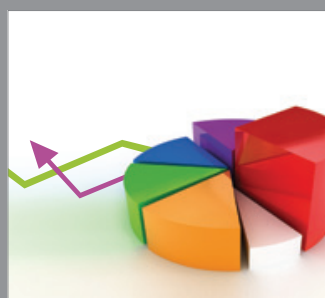

ournal of

Probability and Statistics

Promensencen
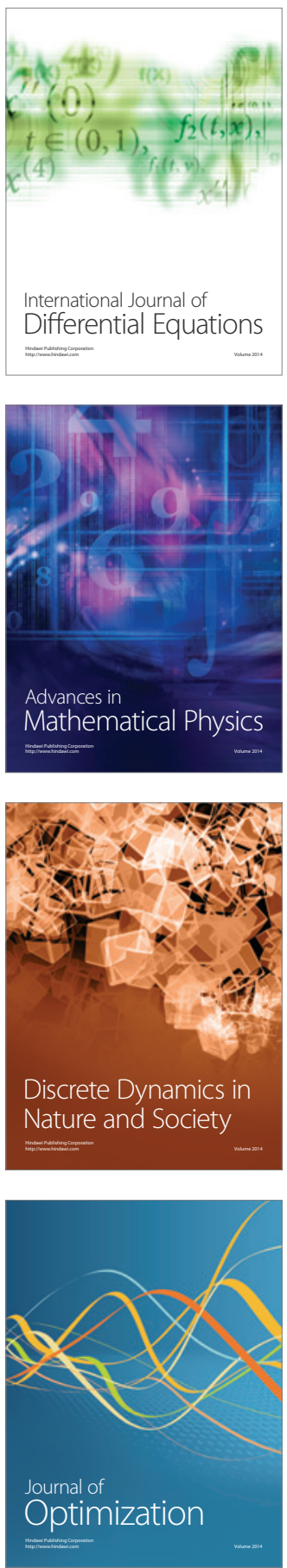